\title{
Material parameter estimation for boron steel from simultaneous cooling and compression experiments
}

\author{
P Åkerström, B Wikman and M Oldenburg \\ Division of Solid Mechanics, Luleå University of Technology, SE-97187 Luleå, Sweden \\ E-mail: Paul.Akerstrom@1tu.se
}

Received 18 April 2005, in final form 3 October 2005

Published 4 November 2005

Online at stacks.iop.org/MSMSE/13/1291

\begin{abstract}
In order to increase the accuracy of numerical simulations of the hot stamping process, reliable material data is crucial. Traditionally, the material is characterized by several isothermal compression or tension tests performed at elevated temperatures and different strain rates. The drawback of the traditional methods is the appearance of unwanted phases for some test temperatures and durations. Such an approach is also both time consuming and expensive. In the present work an alternative approach is proposed, which reduces unwanted phase changes and the number of experiments. The isothermal mechanical response is established through inverse modelling of simultaneous cooling and compression experiments. The estimated material parameters are validated by comparison with data from a separate forming experiment. The computed global response is shown to be in good agreement with the experiments.
\end{abstract}

\section{Introduction}

The use of ultra high strength steel components has increased in the automotive industry, owing to the need for higher passive safety and reduction of weight. Hot stamping of boron alloyed steels is one technique to produce open section, ultra high strength steel components. This manufacturing process is used in a number of vehicle structure applications, for example, side impact protection beams and for bumper system components. The prediction of the product manufacturing and performance requires a detailed knowledge of the material response during the complete manufacturing process, including deformations during the fast cooling from approximately $900{ }^{\circ} \mathrm{C}$ to room temperature. Coupled thermo-mechanical analysis is used to predict the material behaviour during the hot stamping process, see [1].

The reliability of the numerical simulations depends not only on the models and methods used, but also on the accuracy and applicability of the input data. The material model and 
Table 1. Chemical composition for boron 02 in weight per cent.

\begin{tabular}{lllllll}
\hline $\mathrm{C}[\%]$ & $\mathrm{Si}[\%]$ & $\mathrm{Mn}[\%]$ & $\mathrm{P}[\%]$ & $\mathrm{S}[\%]$ & $\mathrm{Cr}[\%]$ & $\mathrm{B}[\%]$ \\
\hline $0.20-0.25$ & $0.20-0.25$ & $1.00-1.30$ & $0-0.025$ & $0-0.015$ & $0.15-0.25$ & $0.0015-0.0050$ \\
\hline
\end{tabular}

the related material property data must be consistent with the conditions of the material in the process of interest. In the case of simultaneous forming and quenching, the material is subjected to a temperature history of heating and subsequent cooling. Since the complete thermo-mechanical history will influence the material properties, similar deformation and temperature histories must be used for the material testing. Traditionally, the material is characterized by several tension, compression or torsion tests at elevated temperatures and different strain rates, see, e.g. [2-5]. One drawback with isothermal tests is the increase of unwanted phases for certain test temperatures and durations. For tests with austenite, the time to perform isothermal testing of boron alloyed steel is limited, see, e.g. [6]. In the temperature interval from $600{ }^{\circ} \mathrm{C}$ to the martensite start temperature at approximately $400{ }^{\circ} \mathrm{C}$, the time allowed for a complete isothermal compression test is less than $10 \mathrm{~s}$ before bainite starts to form. This time for cooling from approximately $900{ }^{\circ} \mathrm{C}$, temperature stabilization and compression is too short for moderate deformation rates.

In the present work an alternative approach is proposed in which unwanted phase transformations are avoided and the number of tests needed can be significantly reduced. The material response during cooling and deformation is studied by inverse modelling of experiments with simultaneous cooling and deformation. With this approach, the mechanical properties are established for a broad temperature range with only a few physical tests. Furthermore, an obvious advantage of the inverse method is also that the inhomogeneous stress and strain conditions in the specimen are taken into account in the evaluation. In [7] a similar technique is used to estimate the material parameters by a single compression test during continuous cooling. The method described here is a development of [7] in order to provide more information for the material characterization process by using several tests.

\section{Experimental procedure}

The compression tests have been carried out in a Gleeble 1500 thermo-mechanical test equipment. The material used is boron steel from Swedish Steel AB-SSAB, see table 1 for chemical composition. The circular cylindrical specimens have the following dimensions: height $5.5 \mathrm{~mm}$ and diameter $4.0 \mathrm{~mm}$. The test procedure can shortly be described by the following steps:

(a) insertion of the specimen into a vacuum chamber,

(b) resistive heating to the austenitic temperature and holding for $300 \mathrm{~s}$,

(c) continuous cooling and compression starting at the desired temperature is maintained until the true axial strain of 0.5 is obtained. The cooling rate and applied axial strain rate are $50{ }^{\circ} \mathrm{C} \mathrm{s}^{-1}$ and $0.08 \mathrm{~s}^{-1}$, respectively.

During a test, the compression force, compression depth, diameter increase and temperature are measured and logged. The diameter increase is measured using a clip-on type water cooled gage with a linear variable displacement transducer (LVDT). The accuracy of the diametric measurements is specified by the manufacturer to be $0.5 \%$ of the full measurement range, $\pm 6 \mathrm{~mm}$, which means that the measurement error is less than $0.06 \mathrm{~mm}$. Direct measurements after the tests with a micrometre caliper give a maximum diameter deviation to the experimental 


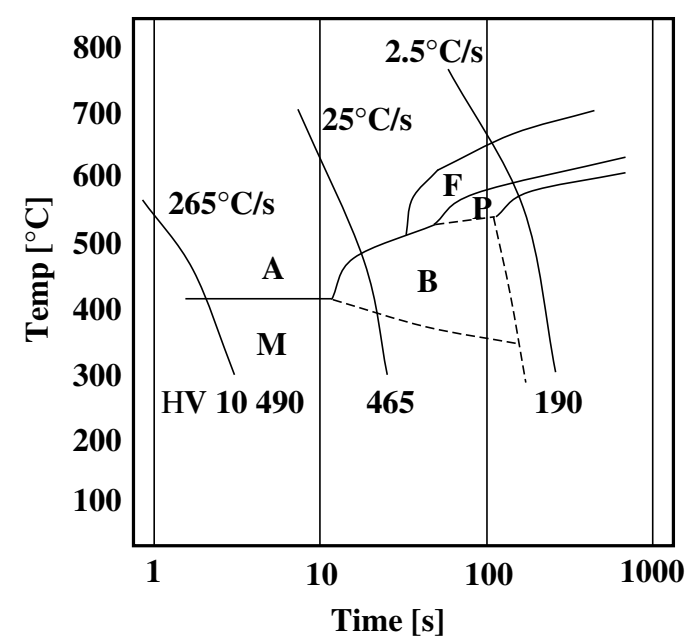

Figure 1. CCT diagram for the boron 02 steel given in the manufacturer's technical specification. The meaning of the letters used in the figure are as follows: A-Austenite, $\mathrm{F}$-Ferrite, $\mathrm{P}$-Pearlite, $\mathrm{B}-$ Bainite and $\mathrm{M}-\mathrm{Martensite}$. The Vickers hardness is denoted by HV.

of $0.05 \mathrm{~mm}$. With the current test equipment, the diametric increase for one cross section at the time can be measured, in this case at the specimen mid-height. The temperature is measured using a Pt/PtRh-thermocouple welded on the surface of the specimen (mid-height). Graphite and tantalum foils have been used to reduce the friction and to prevent sticking between the tungsten carbide anvils and the test specimen. For the strain rate $0.08 \mathrm{~s}^{-1}$, three overlapping temperature intervals for the compression tests have been carried out in order to provide a wide range of deformation-temperature combinations. The temperature intervals for the strain rate of $0.08 \mathrm{~s}^{-1}$ are (in degree centigrade): high-[936,630], medium - [788,481] and low- 638,350$]$. Two specimens at each temperature interval have been tested, to assure that the obtained data are reasonable and that the deviations between the tests are small. The continuous cooling diagram (CCT), which indicates the hardenability of the steel, is shown in figure 1 . The diagram shows that a cooling rate of $50{ }^{\circ} \mathrm{C} \mathrm{s}^{-1}$ would give a final microstructure containing only martensite.

\section{Numerical procedures}

The parameter estimation process is based on the inverse approach, see, e.g. [7-10]. Here the FE-method is applied to solve the equations of motions of the direct problem, see section 3.1, and an optimization algorithm is used to find a suitable set of material parameters to a model describing the material hardening.

\subsection{The direct problem}

The direct problem consists of computing the response of the FE model, which is subjected to variations of the parameters in a model that generates the flow stress. In the present work the explicit FE program LS-Dyna [11] is used for solving the direct problem. An explicit simulation is accomplished without forming a stiffness matrix or solving any equations, thus reducing core storage requirements and avoiding solution failures, see [12]. Complex geometries in industrial applications with contact impact, large deformations, nonlinear materials and 


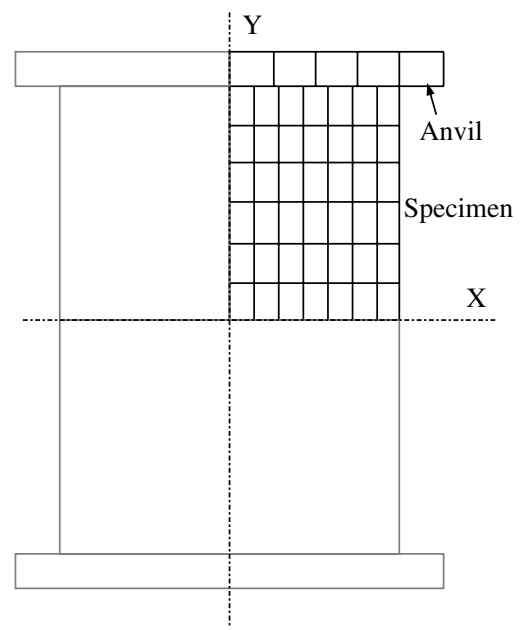

Figure 2. Axisymmetric FE mesh that describes the Gleeble model.

coupled field models can be treated effectively and quickly. Explicit codes are commonly used for large scale coupled thermo-mechanical analyses of metal forming processes in industrial applications.

The material model used is a rate independent thermo-elastic-plastic model described in $[1,13]$. The plastic behaviour is described by a von Mises isotropic yield function, an associated flow rule and a nonlinear isotropic hardening law defined as tabulated values of yield stress and effective plastic strain. The axisymmetric FE mesh used in the simulation of the Gleeble experiments is shown in figure 2. The dimensional increase of the FE specimens due to the resistive pre-heating is not accounted for. The mesh, which is a quarter of the actual geometry due to symmetry conditions, consist of 68 nodes and 47 four-node fully integrated quadrilateral elements (volume weighted Galerkin elements, see [11]). Displacement boundary conditions are applied to the anvil, taken from the experiments. The anvil is modelled as a rigid body. Contact between the anvil and the specimen is included, and the friction is assumed to follow Coulomb's friction law.

During each simulation, the temperature variation within the specimen is neglected and assumed to be prescribed and homogeneous. To check the validity of this assumption, coupled thermo-mechanical simulations have been conducted. The results are given in section 4.2.

The hardening model is a physically based model developed for both bcc and fcc metals by Nemat-Nasser [14]. Most parameters of this model have a clear physical meaning. The athermal and thermally activated flow stress originally defined for commercially pure tantalum and oxygen free, high conductivity (OFHC) copper can be written as

$\sigma_{y}\left(\dot{\bar{\varepsilon}}, \bar{\varepsilon}^{\mathrm{pl}}, T\right)=\sigma^{0}\left(1-\left(-\frac{k T}{G_{0}}\left(\ln \frac{\dot{\bar{\varepsilon}}}{\dot{\bar{\varepsilon}}_{0}}+\ln f\left(\bar{\varepsilon}^{\mathrm{pl}}, T\right)\right)\right)^{1 / q}\right)^{1 / p} f\left(\bar{\varepsilon}^{\mathrm{pl}}, T\right)+\sigma_{a}^{0} g\left(\bar{\varepsilon}^{\mathrm{pl}}, d_{\mathrm{g}}\right)$.

In equation (1), $\dot{\bar{\varepsilon}}$ is the effective strain rate, $\bar{\varepsilon}^{\mathrm{pl}}$ is the effective plastic strain, $T$ is the temperature, $\sigma^{0}$ is an effective stress value to be determined empirically, $k$ is the Boltzman constant and $G_{0}$ is the magnitude of the energy barrier that the dislocations must overcome. $\dot{\bar{\varepsilon}}_{0}$ is a reference strain rate related to the density and the average velocity of mobile dislocations and barrier spacing. In equation (1), the function $f\left(\bar{\varepsilon}^{\mathrm{pl}}, T\right)$ is defined in the sequel for fcc metals, and 
$\sigma_{a}^{0} g\left(\bar{\varepsilon}^{\mathrm{pl}}, d_{\mathrm{g}}\right)$ is the athermal part of the flow stress (depending on density and the distribution of dislocations, approximated by $\bar{\varepsilon}^{\mathrm{pl}}$ and grain size $d_{\mathrm{g}}$ ). The parameters $p$ and $q$ define the shape of the energy barrier. The function represents the ratio of the initial to the current average dislocation spacing. For fcc metals, the dislocation spacing defines the barrier spacing and changes with the dislocation density. As for oxygen free, high conductivity copper (OFHC copper), the following representations are assumed to apply to the boron steel:

$$
f\left(\bar{\varepsilon}^{\mathrm{pl}}, T\right)=1+a(T) \sqrt{\bar{\varepsilon}^{\mathrm{pl}}}
$$

and

$$
a(T)=a_{0}\left(1-\left(\frac{T}{T_{\mathrm{m}}}\right)^{2}\right)
$$

where $T_{\mathrm{m}}$ is the melting temperature and $a_{0}$ depends on the initial average dislocation spacing. Note that it is assumed that the last term in equation $(1), g\left(\bar{\varepsilon}^{\mathrm{pl}}, d_{\mathrm{g}}\right)$, can be approximated by

$$
g\left(\bar{\varepsilon}^{\mathrm{pl}}, d_{\mathrm{g}}\right) \approx\left(\bar{\varepsilon}^{\mathrm{pl}}\right)^{n_{1}} .
$$

The used hardening model assumes that after a change in temperature the new flow stress is reached instantaneously, without any delay in time. Transient conditions may cause a delay of the response in many materials. This phenomenon is reported in, for example, $[15,16]$, where transient strain rate changes of two orders of magnitude is shown to give a delay of the flow stress level before it reaches the stationary value. How the continuous cooling of the specimen affects the instantaneous flow stress is not investigated in this work.

In this model there are 8 material dependent parameters to be determined: $D_{1}=\sigma^{0}, D_{2}=$ $k / G_{0}, D_{3}=\dot{\bar{\varepsilon}}_{0}, D_{4}=a_{0}, D_{5}=q, D_{6}=p, D_{7}=\sigma_{a}^{0}$ and $D_{8}=n_{1}$. The choice of the 8 material parameters follows the description of the hardening model in [14].

\subsection{The inverse problem}

The solution of the direct problem together with the experimental results gives the necessary input data to the optimization problem. In the Gleeble application, the FE simulation provides output data in the form of an axial reaction force and a diametric increase at mid-height (surface) of the specimen as a function of the model parameters and the friction coefficient. In the present study, three different experiments and corresponding numerical simulations are used for the definition of the objective function.

The objective of the inverse problem is to find the parameters used to generate the flow stress hardening function, which gives the best possible match between the FE solutions and the experimental reference data. An optimization approach for estimating the material parameters $D_{i}$ where $i=1, \ldots, M$, is stated as

$$
\min _{D_{i} \in \mathbb{R}^{M}} \varphi_{\text {tot }}\left(D_{i}\right),
$$

where

$$
\varphi_{\mathrm{tot}}\left(D_{i}\right)=\sum_{k=1}^{3} \varphi^{k}\left(D_{i}\right), \quad i=1,2, \ldots, M
$$

and

$$
\varphi^{k}\left(D_{i}\right)=\frac{1}{2} \sum_{j=1}^{N_{k}}\left(\frac{f_{j}^{\exp }-f_{j}^{\mathrm{fem}}\left(D_{i}\right)}{s_{j}^{f} f_{j}^{\exp }}\right)^{2}+\left(\frac{d_{j}^{\exp }-d_{j}^{\mathrm{fem}}\left(D_{i}\right)}{s_{j}^{d} d_{j}^{\exp }}\right)^{2}
$$


Here $f_{j}^{\text {exp }}, f_{j}^{\text {fem }}, d_{j}^{\text {exp }}$ and $d_{j}^{\text {fem }}$ are the experimental and calculated forces and diametric increases for point $j$ on each curve, respectively. The terms $\left(1 / f_{j}^{\exp }\right)$ and $\left(1 / d_{j}^{\exp }\right)$ are normalization or weight factors that are applied to avoid adding terms of different orders of magnitude. The minimum of equation (6) gives the closest match in a least square sense between the $N_{k}$ sample points of each FE solution and corresponding experimental data (index $k$ is used to represent the different temperature intervals). The justification of the least square criterion is based on the hypothesis that the sum of several different contributions will tend to be normally distributed irrespective of the probability distribution of the individual contributions according to the central limit theorem, see e.g. [17]. In the Gleeble tests, the uncertainties associated with the set of measurements are not known in advance, and we assume that all measurements have the same standard deviation $\left(s_{j}^{f}=s_{j}^{d}=s\right)$ and that the model fits well. Then we can proceed by first assigning an arbitrary constant value, in this case $s_{j}^{f}=s_{j}^{d}=s=1$, to all points. When the material parameters are determined by minimizing equation (6), one can proceed by calculating the standard deviation between measured and calculated data ([18]), see section 4.1.

In searching for the minimum value of equation (6), the subplex algorithm proposed by [19] has been used. The subplex algorithm is a direct search method for unconstrained optimization, i.e. there is no need for calculating the gradient of $\varphi^{k}\left(D_{i}\right)$. The subplex method is well suited for optimizing noisy and non-smooth objective functions. Consequently, constraints and error handling can rationally be implemented by means of uncomplicated penalty functions. The focus in this work has been on testing and evaluating models in the context of inverse analysis. There are a number of more efficient candidate algorithms available for finding local and global minima of functions. However, the choice of optimization method here emphasizes ease of implementation, robustness and stability rather than computational efficiency.

The inverse problem is solved by a set of customized subprograms: analyser programs, interface processors and a connecting network. The analyser programs are the FE program with a user defined material subroutine and the optimization software. Interface processors are in-house programs for setting up the correct input format for each analyser program. The connecting network is in this case the Linux operating system, which can be programmed through shell programs to control the data flow and to manage the necessary data communication. Figure 3 shows a schematic structure for the optimization process.

\subsection{Sensitivity and parameter confidence interval estimation}

In this section a procedure for sensitivity and confidence interval estimation is introduced for the different model parameters. The results can be seen in section 4.1 for the estimated parameters with the friction coefficient 0.02 . A more detailed description of the procedure can be seen in, e.g. [20,21].

3.3.1. Sensitivity. An indicator of the quality of the parameter estimation can be obtained from the Gauss-Newton matrix, $\boldsymbol{G}$, which is a first order approximation of the Hessian matrix for $\varphi_{\text {tot }}$. The objective function for temperature interval No $k$ can be written as

$$
\varphi^{k}(\boldsymbol{D})=\frac{1}{2} \sum_{l=1}^{N_{k}}\left(r_{1 l}^{2}(\boldsymbol{D})+r_{2 l}^{2}(\boldsymbol{D})\right)=\frac{1}{2}\left(\boldsymbol{r}_{1}^{\mathrm{T}}(\boldsymbol{D}) \boldsymbol{r}_{1}(\boldsymbol{D})+\boldsymbol{r}_{2}^{\mathrm{T}}(\boldsymbol{D}) \boldsymbol{r}_{2}(\boldsymbol{D})\right),
$$

where

$$
r_{1 l}(\boldsymbol{D})=\left(f_{l}^{\mathrm{exp}}-f_{l}^{\mathrm{fem}}\right) w_{1 l}
$$




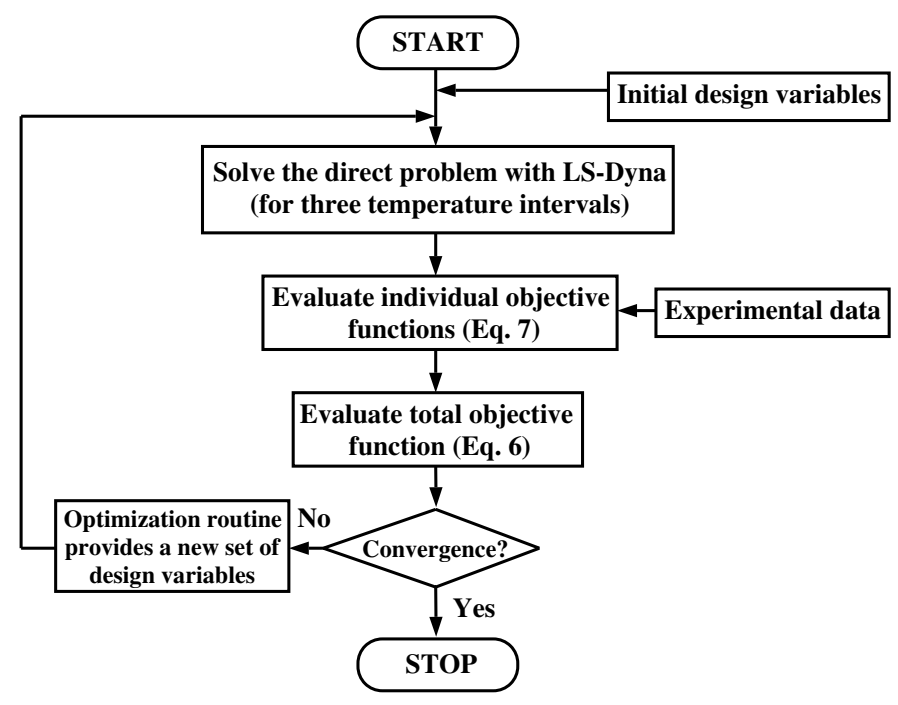

Figure 3. Basic flowchart of the inverse problem.

and

$$
r_{2 l}(\boldsymbol{D})=\left(d_{l}^{\mathrm{exp}}-d_{l}^{\mathrm{fem}}\right) w_{2 l} .
$$

In equation (9) and (10), $w_{1 l}=1 / f_{l}^{\text {exp }}$ and $w_{2 l}=1 / d_{l}^{\text {exp }}$ are the weight factors given in equation (7). The nonlinear objective function is then approximated by a second order Taylor series:

$$
\varphi^{k}(\boldsymbol{D}+\triangle \boldsymbol{D}) \approx \varphi^{k}(\boldsymbol{D})+\frac{\partial \varphi^{k}(\boldsymbol{D})}{\partial \boldsymbol{D}} \Delta \boldsymbol{D}+\frac{1}{2} \Delta \boldsymbol{D}^{\mathrm{T}} \frac{\partial^{2} \varphi^{k}}{\partial \boldsymbol{D}^{2}} \Delta \boldsymbol{D} .
$$

In equation (11) the term $\partial \varphi^{k} / \partial \boldsymbol{D}=\nabla \varphi^{k}$ is the gradient and $\partial^{2} \varphi^{k} / \partial \boldsymbol{D}^{2}=\nabla^{2} \varphi^{k}=\boldsymbol{H}$ is the Hessian. Differentiation of equation (8) gives

$$
\frac{\partial \varphi^{k}(\boldsymbol{D})}{\partial \boldsymbol{D}}=\boldsymbol{J}_{1}^{\mathrm{T}}(\boldsymbol{D}) \boldsymbol{r}_{1}+\boldsymbol{J}_{2}^{\mathrm{T}}(\boldsymbol{D}) \boldsymbol{r}_{2},
$$

where $\boldsymbol{J}$ is the Jacobian matrix that holds the first order partial derivatives. Finally differentiation of the gradient, equation (12), gives the Hessian

$$
\boldsymbol{H}=\boldsymbol{J}_{1}^{\mathrm{T}}(\boldsymbol{D}) \boldsymbol{J}_{1}(\boldsymbol{D})+\boldsymbol{J}_{2}^{\mathrm{T}}(\boldsymbol{D}) \boldsymbol{J}_{2}(\boldsymbol{D})+\boldsymbol{r}_{1}^{\mathrm{T}}(\boldsymbol{D}) \boldsymbol{F}_{1}(\boldsymbol{D})+\boldsymbol{r}_{2}^{\mathrm{T}}(\boldsymbol{D}) \boldsymbol{F}_{2}(\boldsymbol{D}),
$$

where the vector $\boldsymbol{F}$ contains the second order partial derivatives. Since $\boldsymbol{r}$ is minimized in the least square sense, it is often the case that the components of $\boldsymbol{r}$ are small. This makes a first order approximation of the Hessian matrix possible. This is equivalent to making a linear approximation to the residuals $\boldsymbol{r}$. In the Gauss-Newton method, the Hessian is approximated by the first order terms of equation (13) according to

$$
\boldsymbol{H} \approx \boldsymbol{J}_{1}^{\mathrm{T}} \boldsymbol{J}_{1}+\boldsymbol{J}_{2}^{\mathrm{T}} \boldsymbol{J}_{2}=\boldsymbol{G},
$$

where $\boldsymbol{G}$ can be calculated as

$G_{i j}=\left\langle\frac{\partial \varphi_{\mathrm{tot}}}{\partial D_{i}}, \frac{\partial \varphi_{\mathrm{tot}}}{\partial D_{j}}\right\rangle=\sum_{k=1}^{3} \sum_{l=1}^{N_{k}}\left(\left(w_{1 l}\right)^{2} \frac{\partial f_{l}^{\mathrm{fem}}}{\partial D_{i}} \frac{\partial f_{l}^{\mathrm{fem}}}{\partial D_{j}}+\left(w_{2 l}\right)^{2} \frac{\partial d_{l}^{\mathrm{fem}}}{\partial D_{i}} \frac{\partial d_{l}^{\mathrm{fem}}}{\partial D_{j}}\right)_{k}$. 
Here $N_{k}$ is the number of measured points in temperature interval $k$. To approximate the sensitivity terms $\partial f_{l}^{\mathrm{fem}} / \partial D_{m}$ and $\partial d_{l}^{\mathrm{fem}} / \partial D_{m}$ the forward difference approach is applied as

$$
\frac{\partial f_{l}^{\mathrm{fem}}}{\partial D_{m}} \approx \frac{f_{l}^{\mathrm{fem}}\left(D_{m}+\Delta D_{m}\right)-f_{l}^{\mathrm{fem}}\left(D_{m}\right)}{\Delta D_{m}}
$$

and

$$
\frac{\partial r_{l}^{\mathrm{fem}}}{\partial D_{m}} \approx \frac{d_{l}^{\mathrm{fem}}\left(D_{m}+\Delta D_{m}\right)-d_{l}^{\mathrm{fem}}\left(D_{m}\right)}{\Delta D_{m}}
$$

To select appropriate step sizes in equations (16) and (17), a method to determine 'optimal' steps has been used. The procedure is described in [22,23]. An estimation of the correlation between two derivatives can be calculated by

$$
c_{i j}=\cos \left(\frac{\partial \varphi_{\mathrm{tot}}}{\partial D_{i}}, \frac{\partial \varphi_{\mathrm{tot}}}{\partial D_{j}}\right)=\frac{G_{i j}}{\sqrt{G_{i i} G_{j j}}} .
$$

3.3.2. Confidence interval estimation. To estimate confidence intervals for the individual material parameters, the following expressions, see [21], can be used:

$$
I=\left(D_{i}^{\mathrm{opt}}-t \sqrt{V_{i i}}, D_{i}^{\mathrm{opt}}+t \sqrt{V_{i i}}\right),
$$

where the variance can be calculated as

$$
\boldsymbol{V}=\frac{\left[\boldsymbol{G}\left(\boldsymbol{D}^{\mathrm{opt}}\right)\right]^{-1} \varphi_{\mathrm{tot}}\left(\boldsymbol{D}^{\mathrm{opt}}\right)}{N_{\mathrm{tot}}-M}
$$

and $\boldsymbol{G}\left(\boldsymbol{D}^{\text {opt }}\right)$ is the Gauss-Newton matrix calculated in the neighbourhood of the optimal model parameters. $N_{\text {tot }}$ is the total number of points in the measured data and $t$ depends on the confidence level, a 95\% confidence interval gives $t=1.960$.

\subsection{Validation of estimated stress-strain data}

To evaluate the obtained data from the optimization procedure, a separate forming experiment has been carried out. In this section, the experiment and corresponding simulation model are described. Section 4.3.1 summarizes the outcome from the validation.

3.4.1. Validation experiment. The principle of the test is to hot-form a simple hat section with a conventional tool, according to figure 4, from a boron steel strip. The tool material used is SS2242. Prior to forming, the steel strip is heated in an electrical furnace to approximately $930^{\circ} \mathrm{C}$ and kept at this temperature for $300 \mathrm{~s}$. Therefore, we can expect a fully austenitized structure. Figure 5 shows the dimensions of the steel strip. The forming test has been carried out in a Roell Amsler HBS500 test machine. During the test the upper tool was given a constant velocity of $10 \mathrm{~mm} \mathrm{~s}^{-1}$. The process time, forming force, temperature and displacement were recorded during the test. The total time to finish the forming operation was $5 \mathrm{~s}$. Only the force in the actual deformation step is of interest (but not the force when the upper tool hits the bottom and the final cooling and hardening take place). The temperature was measured at several points in the upper/lower tool and at one point on the steel strip edge as illustrated in figure 5 . 


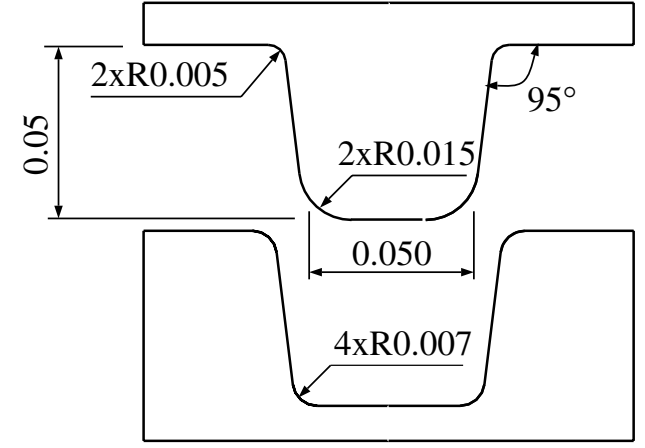

Figure 4. Forming tool that gives a hat section with a profile height of $0.053 \mathrm{~m}$, all dimensions in metres.

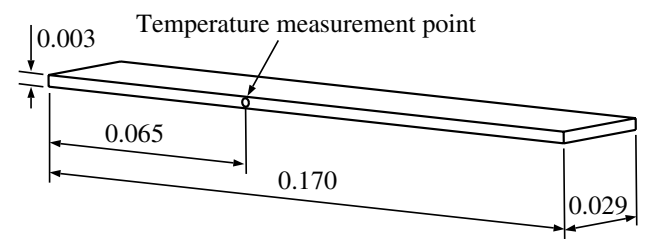

Figure 5. Steel strip used in the experiment, dimensions in metres.

3.4.2. Simulation model. The coupled thermal-mechanical simulation model for LS-Dyna 970 consists of 2746 nodes, 112 shell and 1558 brick elements. The boron steel strip is modelled using four-node Hughes-Liu shell elements, see [11], with 9 through thickness integration points. For the thermal calculations of the steel strip, the shells are treated as twelve-node brick elements to allow heat conduction through the thickness, see [1] for more details. The tool parts are modelled with eight-node brick elements. Only a quarter of the real set-up is modelled due to symmetry. The initial temperature in the steel strip is $827^{\circ} \mathrm{C}$, according to the experiment, and assumed homogeneous. The average temperature, $26^{\circ} \mathrm{C}$, from the experiment measurements is used as a homogeneous initial temperature for the tool. A heat transfer coefficient of $6500 \mathrm{~W} \mathrm{~m}^{-2} \mathrm{~K}^{-1}$ for the tool-sheet interface is used. The gap on each side of the shell element, when thermal contact is considered, is set to $6.0 \times 10^{-5} \mathrm{~m}$. Radiation heat exchange is considered between the surfaces of the strip and its surroundings. The surrounding temperature is assumed to be $30^{\circ} \mathrm{C}$, and the view factor of the strip is set to 1 . An emissivity of 0.6 corresponding to oxidized steel is used. The static and dynamic friction coefficient used between the tool and boron sheet is assumed to be 0.30 . The actual forming time is $5 \mathrm{~s}$. A speed-up factor of 50 is used, which gives a simulated time of $0.01 \mathrm{~s}$. Instead of scaling the thermal conductivity, the heat transfer coefficient and the radiation factor, the specific heat for the tool and boron steel is reduced by a factor $1 / 50$ to compensate for the speed-up.

\section{Results}

When studying the specimens after the test, it can be seen that the bulging effect is quite moderate, indicating that the friction coefficient between the specimen and the foils is low. This is especially prominent for the compression test executed at the lowest temperature interval. For the Nemat-Nasser model, different constant friction coefficients have been tested, and in 


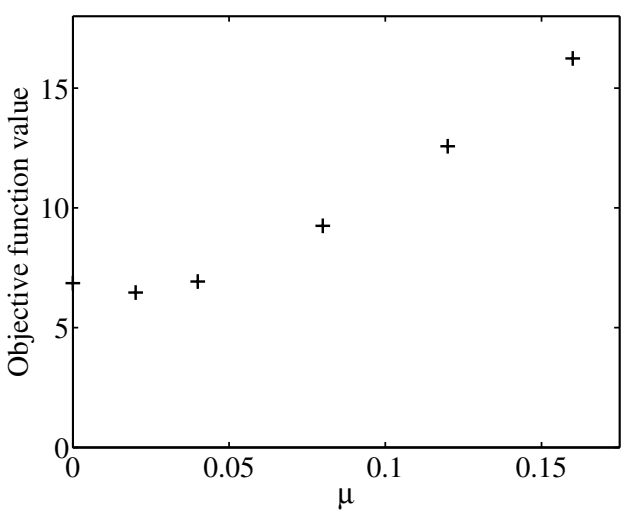

Figure 6. Objective function value for different friction coefficients.

one optimization run the friction coefficient has been included as a design variable. Friction between the tungsten carbide anvils, tantalum foil, graphite foil and the boron specimen in the vacuum chamber at elevated temperatures is not readily obtained. The static and dynamical frictional coefficients are nonlinear relations, dependent on relative sliding velocity, contact pressure, temperature, atmosphere and the nature of the surfaces. In experiments performed by Boyd and Robertson [24] at high pressures (up to $2750 \mathrm{MPa}$ ) and with solid material as lubricants, very low friction coefficients are found. When using graphite as a lubricant [24], a friction coefficient in the interval 0.036-0.058 was obtained. During the Gleeble tests the mean pressure on the specimens contact surfaces reaches $1100 \mathrm{MPa}$. Normally at room temperature and normal atmosphere, the friction coefficient in steel to graphite contact is near 0.1 , according to Bowden and Tabor [25].

In the following sections the terms high, med and low are used in diagram legends to characterize the different temperature intervals as defined in section 2. In the FE-simulation of the Gleeble compression test, the compression time is limited to $5 \mathrm{~s}$ (stop temperature of $389^{\circ} \mathrm{C}$ ) for the low temperature interval to avoid the influence of martensite formation during the cooling/compression cycle.

\subsection{Nemat-Nasser model}

Five different optimization runs with constant coefficients of friction $(0.00,0.04,0.08,0.12$ and 0.16 ) have been conducted. The objective function indicates decreasing values when reducing the friction coefficient. Therefore, an optimization run with the friction coefficient included as an additional design variable has been conducted, resulting in a minima for $\mu=0.02$. Figure 6 shows the objective function value as a function of the friction coefficient.

The friction and material parameters are not independent in the simulation; a variation in the friction coefficient gives a new set of optimal material parameters (and different 'bulging') and therefore, different states of strain and stress within the specimen. The maximum effective plastic strain for the friction coefficients $0.02,0.08$ and 0.16 with corresponding optimized parameters are $0.520,0.616$ and 0.720 , respectively.

The obtained compression forces and diametric increase of the specimen for different friction coefficients together with experimental curves are plotted in figures 7-10. The estimated material parameters are specified in table 2. The effect of the friction variations on the obtained stress-strain curves is illustrated in figure 11 for the temperatures 500 and $800{ }^{\circ} \mathrm{C}$. 

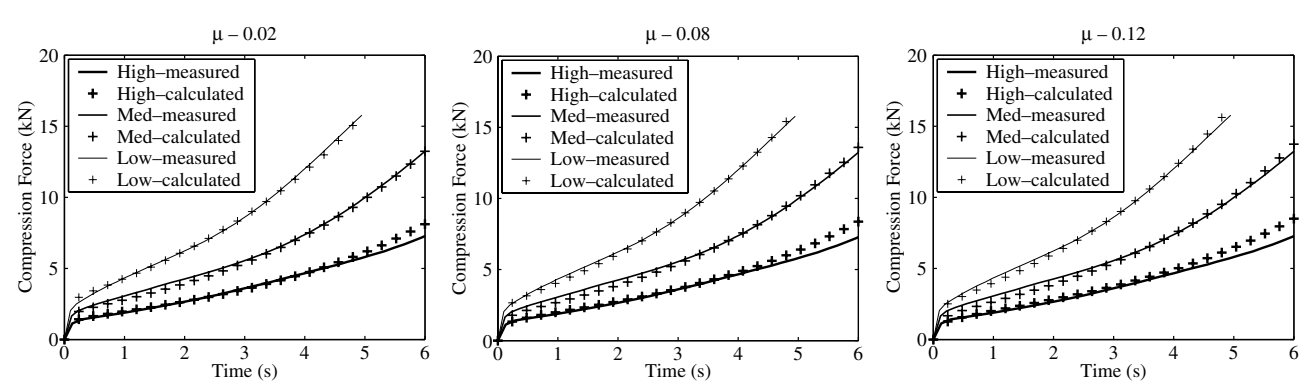

Figure 7. Measured and calculated compression forces for the friction coefficients $(a) \mu=0.02$, (b) $\mu=0.08$ and (c) $\mu=0.12$ used in the simulations.
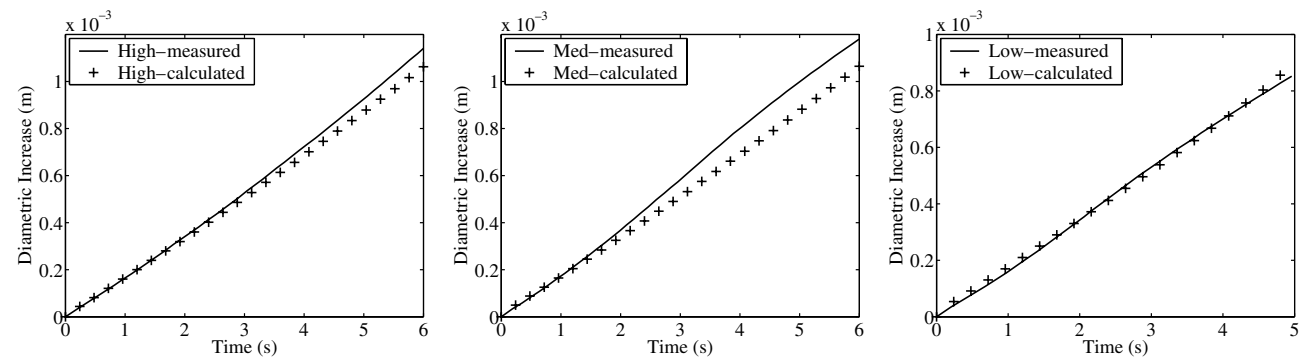

Figure 8. Measured and calculated diametric increase $-\mu=0.02$.
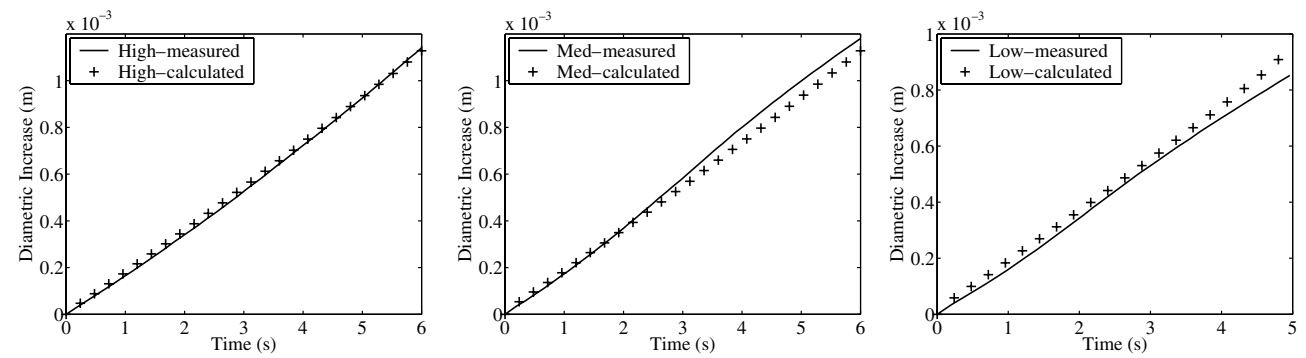

Figure 9. Measured and calculated diametric increase $-\mu=0.08$

Table 3 summarizes the calculated standard deviations of measured and calculated data for the different temperature intervals and friction coefficients. For the actual case, the covariance matrix, $\boldsymbol{c}$, becomes

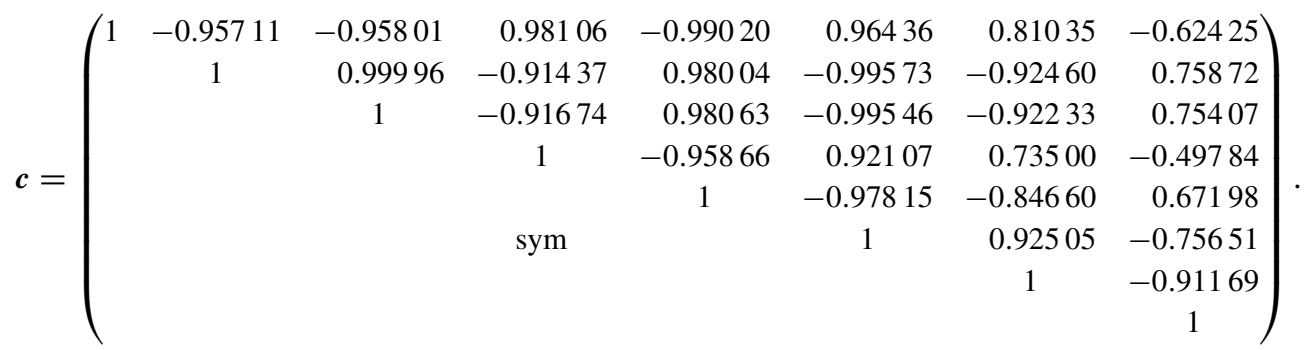



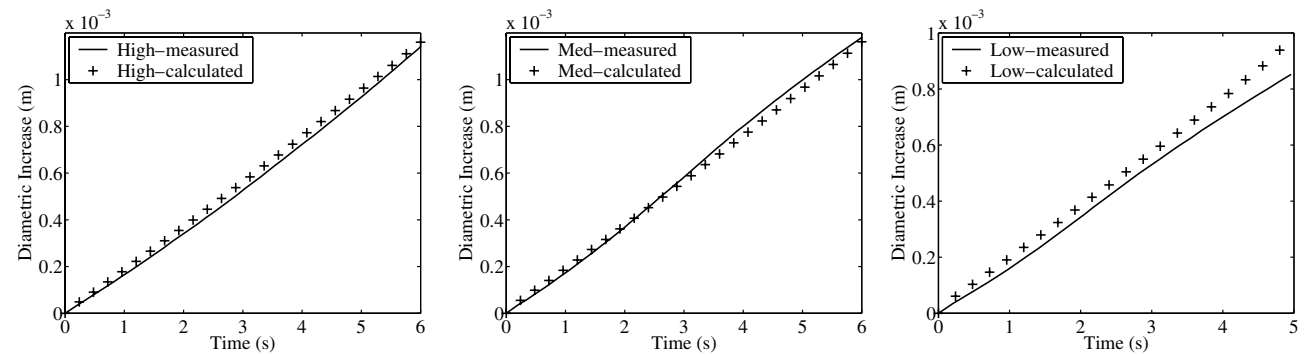

Figure 10. Measured and calculated diametric increase $-\mu=0.12$.

Table 2. Estimated parameters for the Nemat-Nasser model.

\begin{tabular}{lllllll}
\hline$\mu$ & 0.00 & 0.02 & 0.04 & 0.08 & 0.12 & 0.16 \\
\hline$D_{1}=\sigma^{0} 10^{2}[\mathrm{MPa}]$ & 8.445 & 8.885 & 8.396 & 7.483 & 7.760 & 6.818 \\
$D_{2}=k / G_{0} 10^{-5}\left[\mathrm{~K}^{-1}\right]$ & 1.406 & 1.267 & 1.476 & 1.454 & 1.524 & 1.540 \\
$D_{3}=\dot{\bar{\varepsilon}}_{0} 10^{10}\left[\mathrm{~s}^{-1}\right]$ & 2.863 & 0.470 & 3.047 & 2.487 & 2.154 & 3.472 \\
$D_{4}=a_{0}$ & 2.405 & 2.310 & 2.622 & 3.059 & 3.029 & 4.028 \\
$D_{5}=q 10^{-1}$ & 7.598 & 8.008 & 8.464 & 7.829 & 7.714 & 8.049 \\
$D_{6}=p 10^{-2}$ & 7.690 & 6.839 & 10.150 & 8.002 & 7.735 & 8.357 \\
$D_{7}=\sigma_{a}^{0} 10^{2}[\mathrm{MPa}]$ & 1.574 & 1.375 & 1.478 & 1.916 & 2.325 & 2.482 \\
$D_{8}=n_{1} 10^{-1}$ & 1.509 & 1.370 & 1.594 & 2.070 & 2.605 & 2.827 \\
\hline
\end{tabular}

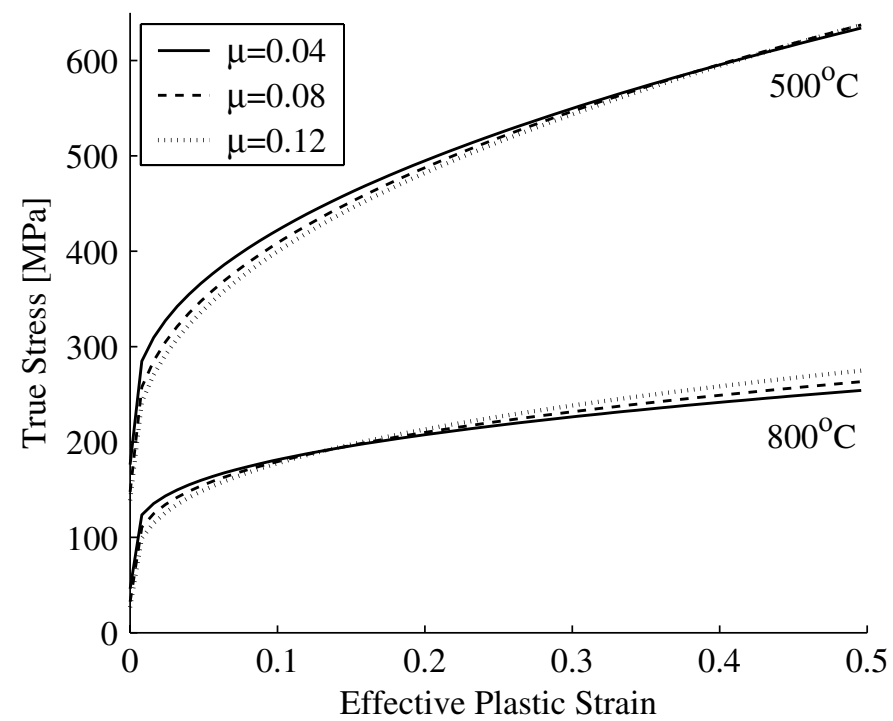

Figure 11. Friction influence on obtained stress-strain curves for the temperatures 500 and $800{ }^{\circ} \mathrm{C}$.

When an off-diagonal element (the cosines between two sensitivity vectors) is close to $\pm 1, G$ is ill conditioned and the confidence intervals become wide. Then the parameters in question are linearly dependent and may be expressed in terms of each other, which means that the influence of two parameters on the data is similar. The three vector combinations that are most 
Table 3. Standard deviations - calculated to measured data for different friction coefficients.

\begin{tabular}{|c|c|c|c|c|c|c|}
\hline \multirow[b]{3}{*}{$\mu$} & \multicolumn{6}{|c|}{ Temperature interval } \\
\hline & \multicolumn{2}{|c|}{ High } & \multicolumn{2}{|c|}{ Medium } & \multicolumn{2}{|c|}{ Low } \\
\hline & $s_{1}^{f}[\mathrm{~N}]$ & $s_{1}^{d} 10^{-5}[\mathrm{~m}]$ & $s_{2}^{f}[\mathrm{~N}]$ & $s_{2}^{d} 10^{-5}[\mathrm{~m}]$ & $s_{3}^{f}[\mathrm{~N}]$ & $s_{3}^{d} 10^{-5}[\mathrm{~m}]$ \\
\hline 0.00 & 267 & 5.25 & 209 & 9.83 & 158 & 1.32 \\
\hline 0.02 & 279 & 3.57 & 196 & 8.22 & 157 & 1.33 \\
\hline 0.04 & 329 & 2.21 & 199 & 6.83 & 147 & 1.95 \\
\hline 0.08 & 387 & 1.39 & 252 & 4.45 & 146 & 3.81 \\
\hline 0.12 & 463 & 2.87 & 297 & 2.64 & 222 & 5.61 \\
\hline 0.16 & 534 & 4.38 & 356 & 1.70 & 324 & 6.89 \\
\hline
\end{tabular}

Table 4. Estimated parameter confidence intervals (95\%).

\begin{tabular}{l}
\hline$D_{1} \in(737.0,1040.0)$ \\
$D_{2} \in\left(-2.763 \times 10^{-7}, 2.561 \times 10^{-5}\right)$ \\
$D_{3} \in\left(-1.097 \times 10^{11}, 1.191 \times 10^{11}\right)$ \\
$D_{4} \in(1.842,2.779)$ \\
$D_{5} \in(0.775,0.826)$ \\
$D_{6} \in(0.063,0.074)$ \\
$D_{7} \in(122.0,153.1)$ \\
$D_{8} \in(0.113,0.162)$ \\
\hline
\end{tabular}

closely correlated (close to \pm 1 ) are

$$
\begin{aligned}
& c_{23}=\cos \left(\frac{\partial \varphi_{\mathrm{tot}}}{\partial D_{2}}, \frac{\partial \varphi_{\mathrm{tot}}}{\partial D_{3}}\right)=0.99996, \\
& c_{26}=\cos \left(\frac{\partial \varphi_{\mathrm{tot}}}{\partial D_{2}}, \frac{\partial \varphi_{\mathrm{tot}}}{\partial D_{6}}\right)=-0.99573,
\end{aligned}
$$

and

$$
c_{36}=\cos \left(\frac{\partial \varphi_{\mathrm{tot}}}{\partial D_{3}}, \frac{\partial \varphi_{\mathrm{tot}}}{\partial D_{6}}\right)=-0.99546,
$$

where $D_{2}=k / G_{0}, D_{3}=\dot{\bar{\varepsilon}}_{0}$ and $D_{6}=p$ according to table 2 . Table 4 shows the estimated parameter intervals.

\subsection{Temperature distribution in the cylindrical specimen}

Coupled thermal-mechanical simulations have been conducted to verify the validity of using a homogeneous temperature assumption. This is shown to only affect the obtained mechanical response marginally as can be seen in figure 12 , making the assumption valid. In these FE simulations the heat transfer coefficient between the anvil and specimen in combination with the temperature of the anvil is adjusted to obtain a temperature drop close to that measured in the experiments. The maximum temperature difference within the specimen for this case is about $30^{\circ} \mathrm{C}$ after $0.5 \mathrm{~s}$ and decreases to $10^{\circ} \mathrm{C}$ at the end of the simulation. Similar temperature differences for the other temperature intervals were obtained. Note that the $30^{\circ} \mathrm{C}$ difference is between the contact interface (top/bottom surface) and the measurement point. At a depth of $0.4 \mathrm{~mm}$ beneath the contact interface, the maximum temperature difference to the measured temperature is $17^{\circ} \mathrm{C}$, decreasing to $7^{\circ} \mathrm{C}$ at the time $6 \mathrm{~s}$. At quarter height, the maximum temperature difference is $7{ }^{\circ} \mathrm{C}$ and is decreasing down to $2{ }^{\circ} \mathrm{C}$ at the time $6 \mathrm{~s}$. A temperature 

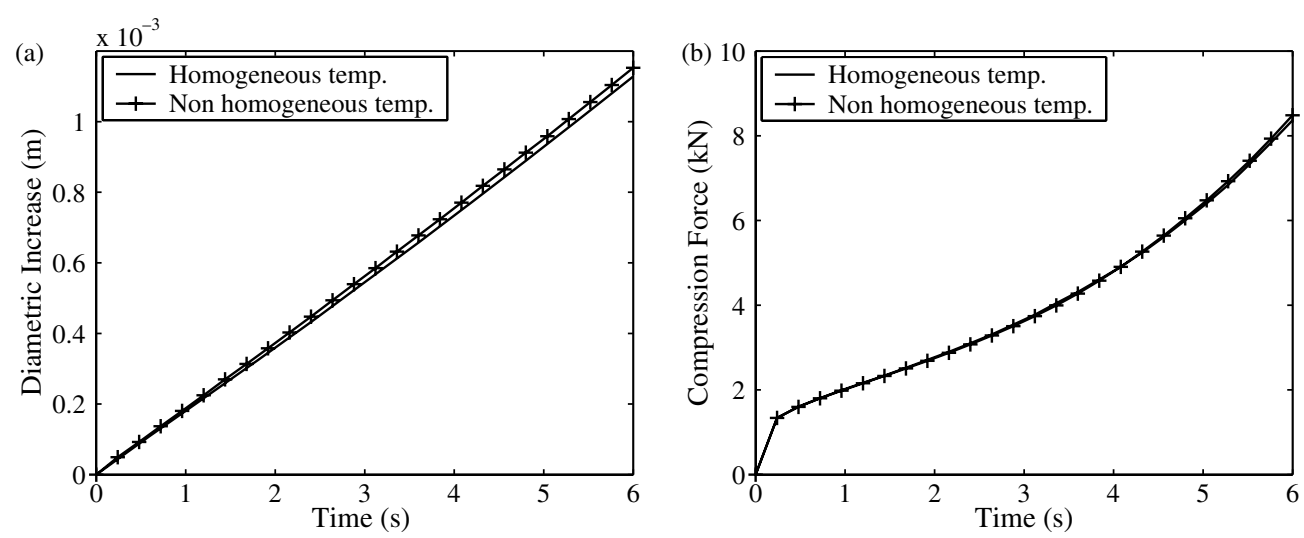

Figure 12. Simulations with and without homogeneous temperature in the specimen; $(a)$ diametric increase of specimen mid (increase from initial diameter), $(b)$ compression force.

decrease of $7{ }^{\circ} \mathrm{C}$ give a rise in flow stress of $1.7 \%$ at $900{ }^{\circ} \mathrm{C}$ and at $600{ }^{\circ} \mathrm{C}$, the corresponding rise is $2 \%$ (using equation (1) with optimized parameters). A homogeneous temperature assumption can therefore be further justified, because a very limited material volume is deviating in temperature more than $7{ }^{\circ} \mathrm{C}$ throughout the experiment. If coupled simulations were applied in the inverse modelling, the obtained temperature history response at the specimen mid-height is not guaranteed to follow the measured, therefore we may introduce errors greater than the above estimated.

\subsection{Strain rate effects}

In this section results from Gleeble compression experiments at higher strain rates are presented together with corresponding FE-simulations. The compression start temperatures correspond to those used for the reference strain rate $0.08 \mathrm{~s}^{-1}$ defined in previous sections. The natural axial strain rates used are 0.5 and $5.0 \mathrm{~s}^{-1}$. In corresponding simulations, the Nemat-Nasser model was used to generate the stress-strain data, based on the estimated parameters. The experimentally measured temperature on each tested specimen is used in the simulation as a prescribed temperature. For the strain rate $0.5 \mathrm{~s}^{-1}$ the mean measured cooling rate is $50-57^{\circ} \mathrm{C} \mathrm{s}^{-1}$. It was not possible to cool the specimens at the strain rate $5.0 \mathrm{~s}^{-1}$. Here an increase in temperature of $1-22^{\circ} \mathrm{C}$ as observed (the highest temperature increase for the lowest start temperature). The rise in temperature is due to plastic deformation. When the rate of heat generation is greater than the heat loss to the anvils and the surroundings, the temperature in the material increases. The material model parameters have been estimated from an experiment using a constant strain rate of $0.08 \mathrm{~s}^{-1}$. This might be unsatisfactory from a completeness point of view. Nevertheless, the model seems to be valid when tested using a forming simulation case, see figure 13. The outcome of the test shows that the model with the optimized parameter set can predict the response for strain rates up to $5 \mathrm{~s}^{-1}$ with good accuracy.

4.3.1. Validation results. Figure 14 shows the measured and calculated temperature histories in the boron steel strip. At the measurement point, the temperature drop was almost $90{ }^{\circ} \mathrm{C}$ during the forming operation, leading to a mean cooling rate of $18^{\circ} \mathrm{C} \mathrm{s}^{-1}$. The maximum mean cooling rate within the sheet reached $63^{\circ} \mathrm{C} \mathrm{s}^{-1}$. Note also that the thermal contact during the forming process was one-sided except when the upper tool approaches its lowest level. Computed and 

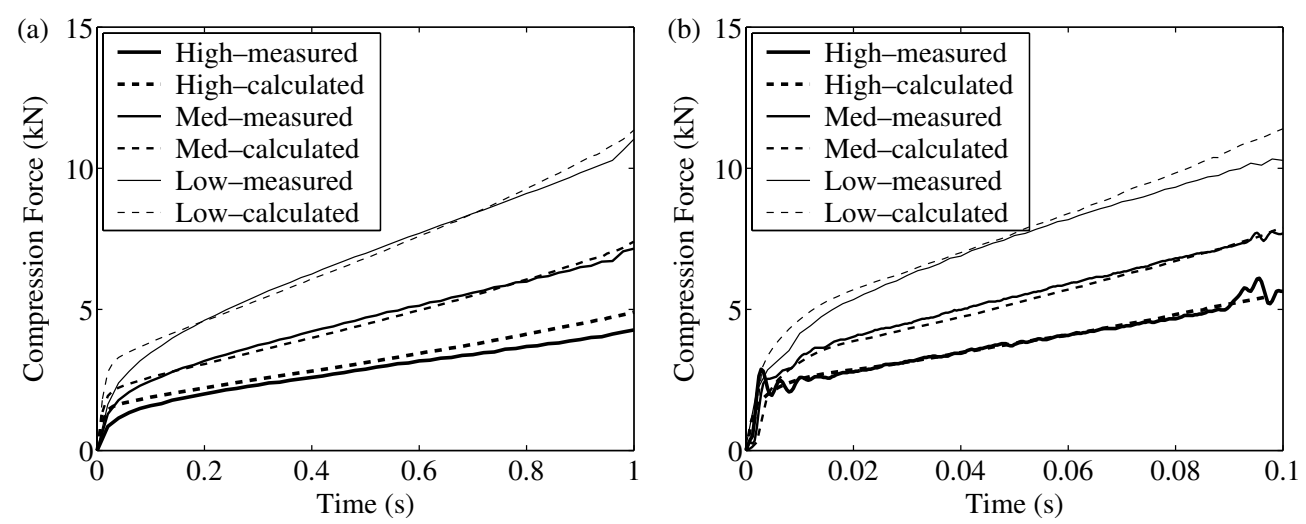

Figure 13. Measured and calculated compression forces at the true axial strain rate of $(a) 0.5 \mathrm{~s}^{-1}$ and $(b) 5.0 \mathrm{~s}^{-1}$.

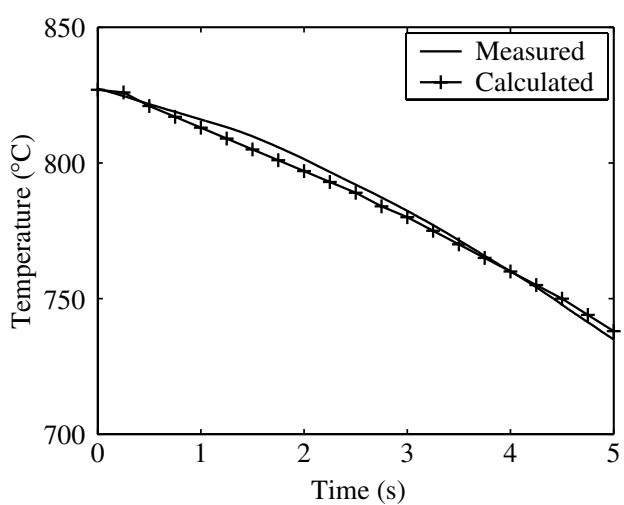

Figure 14. Measured and calculated temperature histories on the boron sheet.

measured forming forces are plotted in figure 15. Note that the global force response is in good agreement with the experimental for the simulation with stress-strain data generated by the optimized parameters for the Nemat-Nasser model (legend NN). For comparison purposes, a simulation with material data obtained from conventional isothermal tension tests is added to figure 15 (legend TD). The obtained forming force in the simulation using tension test data, is at least $20 \%$ lower than the response obtained using the material data from the proposed method. The maximum effective plastic strain in the simulation reached 0.24 slightly below the upper radius. Maximum effective plastic strain at the upper and lower radius reached 0.2 and 0.1 , respectively.

\section{Discussion and conclusions}

In the current paper a methodology for estimation of material parameters for a boron steel grade using thermo-mechanical compression experiments and inverse modelling is presented. Simultaneous cooling and compression experiments are performed instead of traditional isothermal testing. Three different starting temperatures have been considered. The strategy 


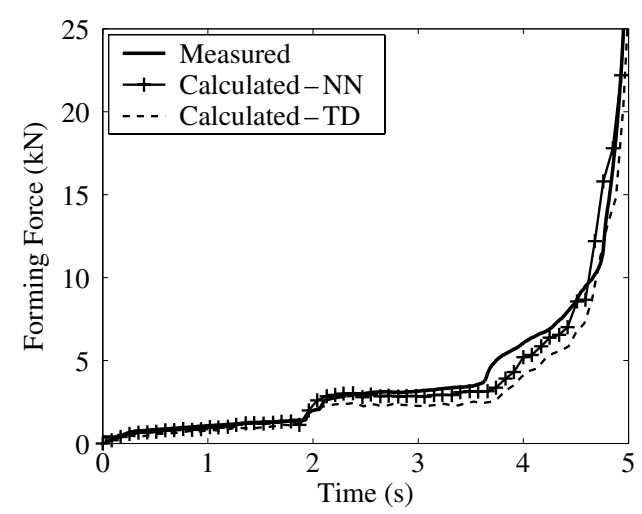

Figure 15. Measured and calculated forming forces in the validation test case $(\mathrm{NN}$-stress strain data from optimization, Nemat-Nasser model; TD—stress strain data from iso-thermal tension tests).

has been to use three overlapping temperature intervals in order to give additionally useful information about the temperature dependent mechanical response.

The objective function is a least square functional, containing the measured reaction forces at the anvils and the mid-specimen diameter change. The choice of a least square criteria has been justified on the basis of the central limit theorem, thus assuming that the sum of several different contributions will tend to be normally distributed, irrespective of the probability of the individual contributions. The least square approach also provides an attractive way of computing the confidence with which the parameters are determined. Note that with the here assumed degree of confidence, parameters $D_{2}$ and $D_{3}$ may take negative values according to table 4. This is, of course, not physically realistic and would corrupt the material model. Nevertheless, it is in agreement with the supposed error distribution. An alternative solution would be to use a non-symmetric distribution instead. In practice this problem is managed by applying physically sound constraints on each parameter. The purpose of the current confidence study is to estimate and picture the uncertainty in the model parameters based on normally distributed errors, not to assert the validity of their values in the interval. Parameter values shall always be examined for their physical soundness before use in simulation models.

One difficulty of the presented method is the influence of friction between the specimen and the anvils during compression. The constant Coulomb friction model used shows that a reduction of the friction coefficient tends to reduce the slope of the flow stress curve. The maximum shift in yield stress is about $40 \mathrm{MPa}$ for a change in the friction coefficient from 0.04 to 0.12 at $500{ }^{\circ} \mathrm{C}$. The corresponding stress shift is $22 \mathrm{MPa}$ at $800^{\circ} \mathrm{C}$. To further improve the proposed method to account for the nonlinear friction dependence on temperature, a similar methodology as described in [26] and shortly summarized below may be employed. In [26] two alternative approaches to estimate the friction coefficient during compression of hollow cylinders (rings) as function of the temperature are presented. In the first approach, the flow stress description is assumed to be independent of the friction coefficient. Then the objective function is formulated as the difference (simulation to experiment) in diameter at several locations on the inner and outer surface of the ring after the experiment to estimate the average of the friction coefficient. The procedure is repeated for three constant temperatures, 800, 900 and $1000{ }^{\circ} \mathrm{C}$. From these results, a linear function is fitted to describe the friction factor as a function of temperature. In approach two, both the difference in measured and calculated compression force and the difference in final shape of the ring are included in the objective 
function. Both the constant in the linear friction model and several material parameters are then included as design variables in the inverse modelling. This last approach is showed to further improve the simulated response.

An interesting extension of the proposed model would be to find a suitable method to continuously measure the specimen diameter at many points along the specimen during compression, not only at the mid-height section as in the current work. It is unclear if the friction coefficient in the current work would be accurately represented by a linear function of temperature due to the wide temperature span or if some more complicated friction law need to be employed.

The uniqueness of the solution has been tested by changing initial values of the parameters. Different minima are found, i.e. the final parameter values change slightly when different initial values are used. However, the parameters reach realistic values and the final objective function value changes negligibly. An explanation to this behaviour can be based on the correlation analysis, which reveals that the optimization problem to some extent is ill conditioned.

With the proposed methods, it is shown that the evaluation of material properties can be performed for conditions where traditional experiments and evaluations would be practically impossible to accomplish.

\section{Acknowledgments}

The financial support from SSAB HardTech AB and the Research Council of Norrbotten are gratefully acknowledged.

\section{References}

[1] Bergman G and Oldenburg M 2004 A finite element model for thermo-mecanical analysis of sheet metal forming. Int. J. Numer. Methods Eng. 59 1167-86

[2] Rao K P, Prasad Y K D V and Hawbolt E B 1996 Hot deformation studies on a low-carbon steel: Part 1-flow curves and the constitutive relationship J. Mater. Process. Technol. 56 897-907

[3] Rao K P, Prasad Y K D V and Hawbolt E B 1996 Hot deformation studies on a low-carbon steel: Part 2-an algorithm for the flow stress determination under varying process conditions J. Mater. Process. Technol. 56 908-17

[4] Kowalski B, Sellars C M and Pietrzyk M 200040 Development of a computer code for the interpretation of results of hot plane strain compression tests ISIJ Int. 40 1230-6

[5] Khoddam S and Hodgson P D 2004 Conversion of the hot torsion test results into flow curve with multiple regimes of hardening J. Mater. Process. Technol. 153-154 839-45

[6] Somani M C, Karjalainen P L, Eriksson M and Oldenburg M 2000 Dimensional changes and microstructural evolution in a b-bearing steel in the simulated forming and quenching process ISIJ Int. 41 361-7

[7] Eriksson M, Wikman B and Bergman G 2003 Estimation of material parameters at elevated temperatures by inverse modelling of a gleeble experiment IUTAM Symp. on Field Analyses for Determination of Material Parameters-Experimental and Numerical Aspects (Abisko National Park, Kiruna, Sweden) ed P Ståhle and K G Sundin (Dordrecht: Kluwer) pp 151-65

[8] Tarantola A 1987 Inverse Problem Theory (Amsterdam: Elsevier)

[9] Mahnken R and Stein E 1994 The identification of parameters for visco-plastic models via finite-element methods and gradient methods Modelling Simul. Mater. Sci. Eng. 2 597-616

[10] Mahnken R and Stein E 1994 Gradient-based methods for parameter identification of viscoplastic materials Inverse Problems in Engineering Mechanics ed M Tanaka et al (Rotterdam: Balkema)

[11] Hallquist J O 1998 LS-DYNA-Theoretical Manual LSTC

[12] Belytschko T 1983 An overview of semidiscretization and time integration procedures Computational Methods for Transient Analysis (Amsterdam: Elsevier) pp 1-65

[13] Bergman G and Oldenburg M 1997 Verification of thermo-mechanical material models by thin plate quenching simulations J. Therm. Stresses 20 679-95 
[14] Nemat-Nasser S 1999 Experimental-based micro mechanical modelling of metal plasticity with homogenisation from micro- to macro-scale properties IUTAM Symp. on Micro- and Macro Structural Aspects of Thermo Plasticity (Bochum, Germany) ed O T Stein and E Stein, pp 101-13

[15] Immarigeon J-P A and Jonas J J 1971 Flow stress and substructural change during the transient deformation of armco iron and silicon steel Acta Metall. 19 1053-61

[16] Urcola J J and Sellars C M 1987 Effect of changing strain rate on stress-strain behaviour during high temperature deformation Acta Metall. 35 2637-47

[17] Kreyszig E 1999 Advanced Engineering Mathemathics 8th edn (New York: Wiley)

[18] Press W H and Flannery B P 1992 Numerical Recipes in Fortran 2nd edn (Cambridge: Cambridge University Press)

[19] Rowan T H 1990 Functional stability analysis of numerical algorithms Doctoral Thesis The University of Texas at Austin, USA

[20] Forestier R, Chastel Y and Massoni E 2002 3d inverse analysis model using semi-analytical differentiation for mechanical parameter estimation 4th Int. Conf. on Inverse Problems in Engineering (Rio de Janerio, Brazil, 2002)

[21] Fletcher R 1980 Practical Methods of Optimization-Unconstrained Optimization vol 1 (New York: Wiley)

[22] Iott J, Haftka R T and Adelman H M 1985 Selecting step sizes in sensitivity analysis by finite differences Technical Memorandum 86382, NASA

[23] Gill P E, Murray W and Saunders M A 1983 Computing forward-difference intervals for numerical optimization SIAM J. Sci. Stat. Comput. 4 310-21

[24] Boyd J and Robertson B P 1945 The friction properties of various lubricants at high pressures Trans. ASME 67 $51-6$

[25] Bowden F P and Tabor D 1986 The Friction and Lubricants of Solids (Oxford: Oxford University Press)

[26] Szyndler D and Pietrzyk M 2001 Identification of parameters in the internal variable constitutive model and friction model for hot forming of steels Simulation of Materials Processing: Theory, Methods and Applications ed K-I Mori (Rotterdam: Balkema) pp 297-302 